\title{
Mechanism of Sugar Signaling in Plants
}

\author{
Stephen K, Beena R*, Manju RV, Viji MM and Roy \\ Stepehn \\ Department of Plant Physiology, Kerala Agricultural University, India \\ *Corresponding Author: Beena Radha, Department of Plant Physiology, Kerala \\ Agricultural University, India.
}

DOI: 10.31080/ASAG.2020.05.0948
Received: January 02, 2021

Published: January 22, 2021

(C) All rights are reserved by Beena Radha., et al.

\begin{abstract}
Plants produce a wide range of sugars that are involved in growth and developmental processes. To coordinate and integrate all these processes plants have developed specific mechanisms. A complex network of metabolic and hormone signaling pathways is present in plants that are intimately linked to diverse sugar responses. One of the mechanisms that has been found to modulate actively with other systems is the 'sugar signaling mechanism'. In order to integrate these changes in the different processes and environmental conditions, plants have developed a mechanism to sense these 'sugar signals'. While hormonal signals are generated at micro-molar concentrations, sugar signals are generated at milli-molar concentrations. The sugar signals modulate gene expression by acting on various kinases and phosphatases. Sugar signals are generated through metabolic processes such as relative concentrations of carbohydrates and other nutrients, through the action of specific enzymes or through various sensors and transporters that are present in the nucleus, cytosol or on the surface of certain organelles.After detection of sugars by sensors, the information is passed on through signal transduction and amplifying cascades, resulting in appropriate responses. Signaling cascades ultimately lead to gene regulation. Sugars regulate gene expression by translating nutrient status to modulate growth and development with available carbohydrate content.
\end{abstract}

Keywords: Sugar Signaling; Plants; Nitrogen

\section{Introduction}

Sugars provide the necessary energy for continued growth and survival. Plants are subjected to metabolic fluxes which change dynamically in response to changing environment. Sugars (carbohydrates) are the central components in coordinating these metabolic fluxes. A complex network of metabolic and hormone signaling pathways is present in plants that are intimately linked to diverse sugar responses. Plant metabolism produces a variety of sugars at different times and locations. In order to integrate these changes in the different processes and environmental conditions, plants have developed a mechanism to sense these 'sugar signals'.

Reduced sink demand or nitrogen limitation leads to feedback inhibition of photosynthetic carbon assimilation. Sugars play a pivotal role in the repression of photosynthetic gene expression. Conversely, in the case of sugar depletion, there is activation of photosynthetic gene expression and increased photosynthetic activity.

Regulation of metabolic activity and cell growth is done mainly through growth factors such as light, temperature, water, nutrients etc. or through hormonal signals. Nutrient signals control several regulatory pathways and cellular processes [15]. Inorganic nutrients (N, S, P, K ions) are taken up from the soil whereas sugars are synthesized using light energy and $\mathrm{CO}_{2}$. Plants must additionally sense the quality and amount of light to produce carbon metabolites [11]. Various factors decide whether these metabolites are stored in source leaves or distributed to sink organs such as young organs, roots or seeds. One such factor is the 'sugar signal' which is the focus of the current review. 
Sugars

Sugars are carbohydrates that are synthesized by the plants through the process of photosynthesis. Examples of sugars in plants are glucose, fructose, sucrose, maltose, mannose, trehalose etc. Photosynthate is transported primarily as sucrose [52]. Sucrose is broken down into glucose and fructose [39]. Biochemical, molecular, and genetic experiments have supported a central role of sugars in plant metabolism, growth, and development [46].

Light and sugars regulate growth activities by a coordinated modulation of gene expression and enzyme activities in both carbohydrate-exporting (source) and carbohydrate-importing (sink) tissues. Sucrose and hexoses (mainly glucose and fructose) are the major sensing-molecules [42]. Hexoses favour cell division, growth and metabolism and expansion, whereas sucrose favours differentiation, maturation and storage. They elicit sugar responses in both source and sink organs [9].

Hexoses tend to have greater signaling potential in promoting organ growth and cell proliferation, while sucrose is typically associated with differentiation and maturation [4]. Relative ratios of hexoses to sucrose are perceived by sucrose metabolic enzymes which coordinate and fine-tune growth during key phases of development [23]. Glucose represses genes involved in photorespiration, fatty acid synthesis and mobilization, and nitrogen metabolism, but activates genes involved in sucrose metabolism, respiration, cell wall and starch biosynthesis, which are usually associated with growth and storage.

Sucrose-induced expression has been reported for genes encoding UDP-glucose pyrophosphorylase, anthocyanin biosynthesis enzymes, and a putative chloroplast glucose-6-phosphate/phosphate translocator [49]. Sucrose reduces the steady state mRNA level corresponding to a proton-sucrose symporter involved in phloem loading. Sucrose-specific signaling pathways were also responsible for the repression of Arabidopsistranscription factor ATB2bZIP [40].

\section{Sugars- Essential roles}

The essential roles of sugars are majorly in the following broad areas - They are the substrates in carbon and energy metabolism. They are the basic building blocks involved in polymer biosynthe- sis. They exhibit hormone like functions as primary messengers in signal transduction and critically regulate gene expression [50]. Sugars regulate gene expression by translating nutrient status to modulate growth and development with available carbohydrate content. Therefore sugar responsive gene regulation reflects carbohydrate abundance or depletion. When there is sugar depletion, the genes involved in photosynthesis, carbohydrate remobilization and export, nitrogen metabolism are up-regulated. In the case of sugar abundance, the sink organ activities such as carbohydrate import, utilization and storage, and starch and anthocyanin biosynthesis are accelerated.

\section{Sugar signals}

While hormonal signals are generated at micromolar concentrations, sugar signals are generated at millimolar concentrations. The sugar signals modulate gene expression by acting on various kinases and phosphatases.Generation of sugar signals may be from the following processes - Carbohydrate concentration and relative ratios to other metabolites. Eg: C:N ratio [12] or flux through sugar specific sensors or transporters [45], sucrose molecules which are generated from sucrose synthase enzyme or glucose molecules generated from hexokinase or invertase. Trehalose-6-phosphate synthesized from Trehalose Phosphate Synthase (TPS) [13] is another source of sugar signals. The action of cell wall invertase coincides with the elevated expression of hexose transporters in some systems. Invertase-derived hexose signals can also markedly alter the expression of genes for both the biosynthesis and sensing of specific hormones (e.g. ABA). Balance between invertase and sucrose synthase activity can alter plant development through differential effects on sugar signaling systems [41].

The various sensors involved in the mechanism of sugar signaling are as follows: i) Hexokinase (HXK) apart from catalysing glucose phosphorylation, also acts as a hexose sensor to transduce signals based on sugar availability [16] ii) RGS1 (Regulator of Gprotein signaling), is a cell surface receptor of extracellular glucose sensing [26]. AtRGS1 related G-protein-coupled glucose signal transduction pathway is one of the three glucose signal transduction pathways in Arabidopsis [18] iii) Extracellular glucose and sucrose can be perceived by G-protein-coupled-receptors (GPCRs) [5] iv) Glucose appears to alter the interaction of RGS1 with GPA1 (G 
$\alpha$ subunit in Arabidopsis) leading to sugar specific signaling events [20] v) In budding yeast, novel hexose transporter-like genes SNF3 and RGT2 encode extracellular glucose sensorsvi) Plant dissacharide transporter SUT2 has been proposed to be a putative sucrose sensor [2]. Sucrose transporter SUT4 has been implicated in controlling circadian gene expression by regulating the export of sucrose from the leaves [6].

\section{Signaling cascades}

After detection of sugars by sensors, the information is passed on through signal transduction and amplifying cascades, resulting in appropriate responses. These responses may be through changes in gene expression or altered enzyme activities. This is achieved through the activity of protein kinases, protein phosphatases and other signal transduction mediators such as $\mathrm{Ca}^{+2}$ and calmodulin. One of the most common mechanisms of signal transduction is phosphorylation and dephosphorylation. 14-3-3 proteins bind specifically to phosphorylated substrates [37]. Thereby, they control enzyme activities, subcellular localization and protein-protein interactions required for signal transduction.

Genetic, cellular and systems analyses have revealed that HXK1 is a pivotal and conserved glucose sensor [7]. It directly mediates transcription regulation. It modulates gene expression and multiple plant hormone-signaling pathways [47]. HXK1 is found intracellularly in the nucleus [8]. Several other plant hexokinases (HXKs) are located on the outer mitochondrial membrane as well as on other cell organelles such as chloroplasts, golgi complexes etc. [22]. KIN 10/11 are energy sensor protein kinases. They function as master regulators of transcription networks under sugar and energy deprivation conditions [36].

The kinase that is specifically inhibited by the antibiotic rapamycin is Target of Rapamycin (TOR). It is a central regulatory hub connecting external signals such as the availability of nutrients or presence of hormones to outputs such as transcription of RNA, translation, ribosome biogenesis, translocation of regulatory proteins, autophagy, storage of reserve compounds [14].

Sucrose non-fermenting Related Kinase (SnRK1) is a plant multi-subunit kinase. SnRK1 is activated by stress conditions that limit energy availability in the cell such as hypoxia, flooding and reduced photosynthetic capacity [55]. SnRK1 represses energy-de- manding processes such as anabolism or cell division. It activates stress-dependant energy generating processes such as starch reallocation and gluconeogenesis [54]. In plants, they play a direct role in carbon metabolism. They phosphorylate and inactivate 3 key biosynthetic enzymes viz. 3-hydroxy-3 methyl glutaryl CoA reductase, nitrate reductase (NR), Sucrose phosphate synthase (SPS). The processes positively promoted by TOR are regulated by SnRK1 in an opposite manner. High sugar levels inhibit SnRK1 [31]. Trehalose-6-phosphate (T6P) which senses sugar availability is a direct negative regulator of SnRK1 activity [57]. SnRK1 phosphorylates and regulates the activity of transcription factor basic leucine zipper 63 (bZIP63) in order to trigger global adaptation to low energy [28].

Trehalose-6-phosphate (T6P) has a role in controlling glycolysis and sugar signaling through its interaction with hexokinase [17]. It modifies the perception of glucose content sensed by HXK. When Trehalose-6-phosphate (T6P) content is higher, a carbon deficit is perceived and photosynthetic capacity is enhanced [33]. Trehalose phosphate synthase (TPS) interacts with 14-3-3 proteins and forms a complex when inactive. Under carbon starvation conditions, the complexes undergo dissociation to liberate Trehalose-6-phosphate (T6P) to interact with enzymes and proteins such as hexokinase.

\section{Sugar functions}

Sugars regulate diverse processes in plants. They control cell division through modulation of cyclin D gene expression [24]. Sugars cross-talk with phytohormone-signaling networks to modulate critical growth processes such as embryo establishment, seed germination, and seedling and tuber growth $[43,51]$. They have also been reported to regulate genes that control meristem maintenance and identity [44]. Pien., et al. [35] observed spatio temporal expression of carbohydrate metabolic genes in the tomato shoot apical meristem (SAM) and developing leaf primordial. Sugar signaling interacts with hormone, light and stress signaling by regulating the expression of diverse pathway components and transcription factors. Circadian clock allows plants to anticipate daily changes in its metabolism. This is achieved by the sensing of quality, quantity of light integrated with the level of sugar production ensuring an appropriate response of metabolism to specific situations [6]. Several nitrogen-regulated genes are co-regulated by sugars [10]. Plants display photosynthesis, respiration and fermentation at the same 
time in different tissues. This is possible through a complex regulatory system that involves sugar signaling that integrates different metabolic, developmental and environmental signals to control metabolic rate and activity. Sugars regulate the expression of wound inducible proteinase inhibitor II and lipoxygenase genes, pathogenesis related (PR) genes and dark-inducible (DIN) genes [43]. Mishra., et al. [30] reported that glucose regulated $62 \%$ of the genes that were affected by auxin. Many jasmonate, ABA and stress inducible genes are co-regulated by sugars. Photosynthesis is controlled at the molecular level by sugars and nitrogen signals through changes in the whole plant carbon-nitrogen balance. This overrides the control of photosynthesis by other mechanisms [34].

\section{Cross-talk}

Sugars have been found to be interacting extensively with nutrient, environmental, hormonal factors. Under high temperature conditions, anther abortion was noted as a consequence of disturbance in sugar metabolism which influenced the auxin and cytokinin concentrations in cotton [29]. Plants defective in abscicic acid (ABA) and/or ethylene perception and signaling tend to display altered sugar response pathways. Sugars and ABA act synergistically during embryo growth. They cause transition from a phase of cell division to cell enlargement leading to accumulation of storage reserves. ABA enhances sucrose induction of starch biosynthetic genes [38]. ABA and glucose act antagonistically during seed germination and early seedling growth. Exogenous glucose enabled wild-type Arabidopsis seeds to germinate even when ABA concentration level was inhibitory [25].

Plants and pathogens compete for sugars, in which case sugar signals play a regulatory role in the allocation of resources. Their response decides the susceptibility or resistance nature of the plant variety [21]. Microbial pathogens can synthesize and release trehalose to the plant cell. This interferes with the host sugarsensing pathways, thereby manipulating host metabolism in their favour [32].

Multiple regulatory components isolated in any particular signaling pathway have been found to interconnect with other path- ways. This plasticity facilitates central nodes of integration that are crucial for a plant's flexible responses to physical and chemical environments. Transcription factors such as ABI4 andABI5 could act as integration nodes, receiving signals from multiple sources and generating plant responses [25].

\section{Applications}

Several researchers have attempted to manipulate the components involved in the sugar signaling mechanism. Lugassi., et al. [27] genetically modified citrus plants to express Arabidopsis HXK1 (AtHXK1) under KST1, a guard cell-specific promoter. The expression of AtHXK1 in the guard cells of citrus reduced stomatal conductance and transpiration with no negative effect on the rate of photosynthesis, leading to increased water-use efficiency.In another study, it was noted that elevation of CWIN (Cell wall invertase) activity increased rice grain size [50]. Tomato fruit hexose levels were also increased leading to leaf longevity [19]. Suppression of VIN (Vacuolarinvertase) in potato reduced sprouting and disease infection during storage [3]. Over-expression of Sus (Sucrose synthase) in cotton alleviated seed abortion [56]. Over-expression of AGPase in wheat increased grain weight and number [48]. Suppressing Sus expression in guard cells of potato leaves reduced leaf stomata conductance and increased water use efficiency [1]. Overexpression of the tonoplast monosaccharide transporter TMT1 lowers cytosolic glucose level, probably in both source and sink. This leads to enhanced sucrose export from leaf phloem and high seed yield, indicating plasticity in assimilate transport and utilization [53].

\section{Conclusion}

The sugar signaling pathway is not a linear pathway. It is a complex web that interacts dynamically with other signaling pathways. This is done through common nodal points. They integrate both intrinsic and extrinsic signals providing the plasticity with which the plant functions in the changing day to day conditions.Genetic modification of sugar metabolism and transport could alter cellular sugar levels or fluxes. Regulating the enzymes or transporters involved in the sugar signaling pathway could be useful in improving crop 
productivity, modifying the plant developmental processes or developing crop varieties that can tolerate biotic and abiotic stresses. Much progress has been made in understanding and utilising the components of the sugar signaling mechanism. However several components still remain to be elucidated.

\section{Bibliography}

1. Antunes Werner C., et al. "Changes in stomatal function and water use efficiency in potato plants with altered sucrolytic activity". Plant, Cell and Environment 35.4 (2012): 747-759.

2. Barker Laurence., et al. "SUT2, a putative sucrose sensor in sieve elements". The Plant Cell 12.7 (2000): 1153-1164.

3. Bhaskar Pudota B., et al. "Suppression of the vacuolarinvertase gene prevents cold-induced sweetening in potato". Plant Physiology 154.2 (2010): 939-948.

4. Borisjuk Ljudmilla., et al. "Spatial analysis of plant metabolism: sucrose imaging within Viciafaba cotyledons reveals specific developmental patterns". The Plant Journal 29.4 (2002): 521-530.

5. Chandrashekar Jayaram., et al. "The receptors and cells for mammalian taste”. Nature 444.7117 (2006): 288-294.

6. Chincinska Izabela., et al. "Photoperiodic regulation of the sucrose transporter StSUT4 affects the expression of circadianregulated genes and ethylene production". Frontiers in Plant Science 4 (2013): 26.

7. Cho Jung-Il., et al. "Role of the rice hexokinases OsHXK5 and OsHXK6 as glucose sensors". Plant Physiology 149.2 (2009): 745-759.

8. Cho Young-Hee., et al. "Regulatory functions of nuclear hexokinase1 complex in glucose signaling". Cell 127.3 (2006): 579589.

9. Ciereszko Iwona. "Regulatory roles of sugars in plant growth and development". Acta Societatis Botanicorum Poloniae 87.2 (2018).
10. Coruzzi Gloria and Daniel R Bush. "Nitrogen and carbon nutrient and metabolite signaling in plants". Plant Physiology 125.1 (2001): 61-64.

11. Dobrenel Thomas., et al. "TOR signaling and nutrient sensing". Annual Review of Plant Biology 67 (2016): 261-285.

12. Eveland, Andrea L and David P Jackson. "Sugars, signalling, and plant development". Journal of Experimental Botany 63.9 (2012): 3367-3377.

13. Figueroa Carlos $\mathrm{M}$ and John E Lunn. "A tale of two sugars: trehalose 6-phosphate and sucrose". Plant Physiology 172.1 (2016): 7-27.

14. Fonseca Bruno D., et al. "The ever-evolving role of mTOR in translation". Seminars in Cell and Developmental Biology 36 (2014).

15. Francis Dennis and Nigel G Halford. "Nutrient sensing in plant meristems". Plant Molecular Biology 60.6 (2006): 981-993.

16. Granot David., et al. "Hexose kinases and their role in sugarsensing and plant development". Frontiers in Plant Science 4 (2013): 44.

17. Griffiths Cara A., et al. "Chemical intervention in plant sugar signalling increases yield and resilience". Nature 540.7634 (2016): 574-578.

18. Hellmann Hanjo A and SjefSmeekens. "Sugar sensing and signaling in plants". Frontiers in Plant Science 5 (2014): 113.

19. Jiao Yue., et al. "Glucose signaling, AtRGS1 and plant autophagy". Plant Signaling and Behavior 14.7 (2019): 1607465.

20. Jin Ye., et al. "Posttranslational elevation of cell wall invertase activity by silencing its inhibitor in tomato delays leaf senescence and increases seed weight and fruit hexose level". The Plant Cell 21.7 (2009): 2072-2089.

21. Johnston Christopher A., et al. "GTPase acceleration as the rate-limiting step in Arabidopsis G protein-coupled sugar signaling". Proceedings of the National Academy of Sciences 104.44 (2007): 17317-17322. 
22. Kanwar Poonam and GopaljeeJha. "Alterations in plant sugar metabolism: signatory of pathogen attack". Planta 249.2 (2019): 305-318.

23. Karve Abhijit., et al. "Expression and evolutionary features of the hexokinase gene family in Arabidopsis". Planta 228.3 (2008): 411.

24. Koch Karen. "Sucrose metabolism: regulatory mechanisms and pivotal roles in sugar sensing and plant development". Current Opinion in Plant Biology 7.3 (2004): 235-246.

25. Lastdrager Jeroen., et al. "Sugar signals and the control of plant growth and development". Journal of Experimental Botany 65.3 (2014): 799-807.

26. León Patricia and Jen Sheen. "Sugar and hormone connections". Trends in Plant Science 8.3 (2003): 110-116.

27. Li Lei and Jen Sheen. "Dynamic and diverse sugar signaling”. Current Opinion in Plant Biology 33 (2016): 116-125.

28. Lugassi Nitsan., et al. "Expression of Arabidopsis hexokinase in citrus guard cells controls stomatal aperture and reduces transpiration". Frontiers in Plant Science 6 (2015): 1114.

29. Mair Andrea., et al. "SnRK1-triggered switch of bZIP63 dimerization mediates the low-energy response in plants". Elife 4 (2015): e05828.

30. Min Ling., et al. "Sugar and auxinsignaling pathways respond to high-temperature stress during anther development as revealed by transcript profiling analysis in cotton". Plant Physiology 164.3 (2014): 1293-1308.

31. Mishra Bhuwaneshwar S., et al. "Glucose and auxinsignaling interaction in controlling Arabidopsis thaliana seedlings root growth and development". PLOS One 4.2 (2009): e4502.

32. Nunes Cátia., et al. "Inhibition of SnRK1 by metabolites: tissue-dependent effects and cooperative inhibition by glucose 1-phosphate in combination with trehalose 6-phosphate". Plant Physiology and Biochemistry 63 (2013): 89-98.
33. O'Hara Liam E., et al. "How do sugars regulate plant growth and development? New insight into the role of trehalose6-phosphate". Molecular Plant 6.2 (2013): 261-274.

34. Paul Matthew J., et al. "The role of trehalose 6-phosphate in crop yield and resilience". Plant Physiology 177.1 (2018): 1223.

35. Paul Matthew., et al. "Enhancing photosynthesis with sugar signals". Trends in Plant Science 6.5 (2001): 197-200.

36. Pien Stéphane., et al. "Novel marker genes for early leaf development indicate spatial regulation of carbohydrate metabolism within the apical meristem". The Plant Journal 25.6 (2001): 663-674.

37. Ramon Matthew., et al. "Sugar sensing and signaling". The Arabidopsis book/American Society of Plant Biologists 6 (2008).

38. Rolland Filip., et al. "Sugar sensing and signaling in plants". The Plant Cell 14 (2002): S185-S205.

39. Rook Fred., et al. "Impaired sucrose-induction mutants reveal the modulation of sugar-induced starch biosynthetic gene expression by abscisic acid signalling". The Plant Journal 26.4 (2001): 421-433.

40. Rook Fred., et al. "Sucrose-specific signalling represses translation of the Arabidopsis ATB2bZIP transcription factor gene". The Plant Journal 15.2 (1998): 253-263.

41. Rosa Mariana., et al. "Soluble sugars: Metabolism, sensing and abiotic stress: A complex network in the life of plants". Plant Signaling and Behavior 4.5 (2009): 388-393.

42. Ruan Yong-Ling. "Sucrose metabolism: gateway to diverse carbon use and sugar signaling". Annual Review of Plant Biology 65 (2014): 33-67.

43. Sadka Avi., et al. "Phosphate modulates transcription of soybean VspB and other sugar-inducible genes". The Plant Cell 6.5 (1994): 737-749. 
44. Sami Fareen., et al. "Interaction of glucose and phytohormonesignaling in plants". Plant Physiology and Biochemistry 135 (2019): 119-126.

45. Satoh-Nagasawa Namiko., et al. "A trehalose metabolic enzyme controls inflorescence architecture in maize". Nature 441.7090 (2006): 227-230.

46. Smeekens Sjef., et al. "Sugar signals and molecular networks controlling plant growth". Current Opinion in Plant Biology 13.3 (2010): 273-278.

47. Smeekens Sjef. "Sugar-induced signal transduction in plants". Annual Review of Plant Biology 51.1 (2000): 49-81.

48. Smidansky Eric D., et al. "Enhanced ADP-glucose pyrophosphorylase activity in wheat endosperm increases seed yield". Proceedings of the National Academy of Sciences 99.3 (2002): 1724-1729.

49. Solfanelli Cinzia., et al. "Sucrose-specific induction of the anthocyanin biosynthetic pathway in Arabidopsis". Plant physiology 140.2 (2006): 637-646.

50. Thompson Michael., et al. "Effects of elevated carbon dioxide on photosynthesis and carbon partitioning: a perspective on root sugar sensing and hormonal crosstalk". Frontiers in Physiology 8 (2017): 578.

51. Wang Ertao., et al. "Control of rice grain-filling and yield by a gene with a potential signature of domestication". Nature Genetics 40.11 (2008): 1370-1374.

52. Wang, Lu and Yong-Ling Ruan. "Shoot-root carbon allocation, sugar signalling and their coupling with nitrogen uptake and assimilation". Functional Plant Biology 43.2 (2016): 105-113.

53. Wingenter Karina., et al. "Increased activity of the vacuolar monosaccharide transporter TMT1 alters cellular sugar partitioning, sugar signaling, and seed yield in Arabidopsis". Plant Physiology 154.2 (2010): 665-677.

54. Wingler Astrid. "Transitioning to the next phase: the role of sugar signaling throughout the plant life cycle". Plant Physiology 176.2 (2018): 1075-1084.
55. Wurzinger Bernhard., et al. "The SnRK1 kinase as central mediator of energy signaling between different organelles". Plant Physiology 176.2 (2018): 1085-1094.

56. Xu Shou-Min., et al. "Overexpression of a potato sucrose synthase gene in cotton accelerates leaf expansion, reduces seed abortion, and enhances fiber production". Molecular Plant 5.2 (2012): 430-441.

57. Zhang Yuhua., et al. "Inhibition of SNF1-related protein kinase 1 activity and regulation of metabolic pathways by trehalose-6-phosphate". Plant Physiology 149.4 (2009): 1860-1871.

\section{Assets from publication with us}

- Prompt Acknowledgement after receiving the article

- Thorough Double blinded peer review

- Rapid Publication

- Issue of Publication Certificate

- High visibility of your Published work

Website: www.actascientific.com/

Submit Article: www.actascientific.com/submission.php

Email us: editor@actascientific.com

Contact us: +919182824667 